\title{
Multi-session fecal microbiota transplantation using colonoscopy has favorable outcomes for the treatment of steroid-dependent ulcerative colitis
}

\author{
Young-Seok Cho \\ Division of Gastroenterology, Department of Internal Medicine, Seoul St. Mary's Hospital, College of Medicine, The Catholic University of Korea, \\ Seoul, Korea
}
Article: Efficacy of fecal microbiota therapy in steroid dependent ulcerative colitis: a real world intention-to-treat analysis (Intest Res 2019;17:78-86)

The pathogenesis of IBD remains unclear; however, it is generally accepted that IBD is caused by aberrant immune responses toward antigens derived from commensal bacteria in genetically susceptible individuals. Moreover, consistent alterations in the gut microbiota have been repeatedly observed in IBD, and now are recognized as a key contributing factor to its pathogenesis. ${ }^{1}$ An imbalance in gut microbiota, known as "dysbiosis" in IBD, is characterized by reduced microbial diversity, with a notable decrease in the Bacteroides phylum and Lachnospiraceae group of the phylum Firmicutes, and an increase in Proteobacteria. ${ }^{2}$ However, it is still unclear whether dysbiosis itself causes IBD or whether it represents an epiphenomenon of microbial alterations as a consequence of the disease. ${ }^{3}$ Current treatment for modifying the gut microbiome includes antibiotics, probiotics, and prebiotics; however, evidence of their therapeutic benefit in IBD is limited. ${ }^{2}$ Therapeutic success of fecal microbiota transplantation (FMT) for recurrent Clostridium difficile infection (CDI) has attracted great interest in the

Received December 24, 2018. Accepted December 29, 2018.

Correspondence to Young-Seok Cho, Division of Gastroenterology,

Department of Internal Medicine, Seoul St. Mary's Hospital, College of

Medicine, The Catholic University of Korea, 222 Banpo-daero, Seocho-gu,

Seoul 06591, Korea. Tel: +82-2-2258-6021, Fax: +82-2-2258-2038,

E-mail: yscho@catholic.ac.kr

ORCID Young-Seok Cho (https://orcid.org/0000-0003-1537-3427) use of FMT for various colonic disorders, including IBD such as UC. ${ }^{4}$ Recent randomized controlled trials (RCTs) for the application of FMT in IBD have focused on UC, which might be simpler in terms of objective clinical metrics, compared with CD. ${ }^{3}$ Four RCTs about FMT in adult patients with UC have been published. Although these RCTs have significant heterogeneity with different methods of stool preparation, routes of administration, doses, and selection and preparation of the patient, RCTs and meta-analysis suggest that short-term use of FMT might be beneficial for clinical and endoscopic improvement in mild to moderate UC patients. ${ }^{4-8}$ However, before FMT can be recommended as a routine therapeutic practice, further studies are necessary to define the optimal timing, optimal dosing frequency, route of delivery, role of antibiotic preconditioning, and donor selection for each patient, as well as its longterm safety and efficacy as a maintenance therapy. ${ }^{3}$

In this issue of Intestinal Research, Sood et al. ${ }^{9}$ published the therapeutic outcomes of multi-session FMT over 22 weeks via colonoscopy, in patients with steroid dependent UC. The study results showed a better outcome compared to previous 4 RCTs. At week 24, steroid-free clinical remission, clinical response, and endoscopic remission was $46.3 \%, 75.6 \%$, and $63.4 \%$ respectively. Better responses might be attributed to the route of administration and dosing frequency. The 4 RCTs varied in terms of the FMT infusion protocol, especially the route of adminis- 
tration and frequency of infusions. Moayyedi et al. ${ }^{5}$ administered weekly FMT or placebo enemas for 6 weeks. Rossen et al. ${ }^{6}$ administered FMT or placebo (autologous stool) via a nasoduodenal tube at time zero and 3 weeks. Paramsothy et al. ${ }^{8}$ were more aggressive, administering FMT or placebo by colonoscopy at time zero and via enemas 5 times per week for 8 weeks. In the RCT by Costello et al., ${ }^{7}$ FMT or placebo (autologous stool) was administered at time zero, followed by 2 enemas by day 7. In a study by Sood et al., ${ }^{9}$ patients received the first 2 FMT sessions fortnightly and then every 4 weeks for 22 weeks by colonoscopy, although there was no control arm to compare the efficacy of FMT.

As UC primarily involves the colon and rectum, FMT through the upper GI tract might neutralize the transplanted microbiota before it arrives into the colon. In patients with pancolitis, FMT via enema might be insufficient to cover the entire colon. ${ }^{2}$ Therefore, all FMT sessions by colonoscopy might have resulted in better outcomes. Although the dosing frequency was not very intense in the study by Sood et al., ${ }^{9}$ the rate of steroid-free remission was comparable to that from a more intensive FMT protocol. ${ }^{3,8}$ In contrast to the 4 RCTs and the study by Sood et al., a Japanese study administered a single FMT by colonoscopy in 10 patients with active UC. Although no serious adverse effects were observed, only 1 patient achieved clinical response and none of the patients' microbiota diversity recovered to the donor levels. ${ }^{10}$ Considered together, these results suggest that single FMT might be efficacious for CDI, but not for IBD, which is a more complicated and heterogeneous disease with complex interactions between the genetic, environmental, immunologic, and gut microbial factors. ${ }^{1}$

Another important consideration is selecting the best time to perform FMT during the course of the disease. Moayyedi et al. ${ }^{5}$ showed better outcomes in newly diagnosed UC patients, which suggests a potential window of opportunity to treat patients with FMT, early after diagnosis. In addition, whether FMT can be performed in patients with acute flare-up of UC needs to be considered. As inflammation itself plays an important role in dysbiosis, an active inflammatory state could change the transplanted microbiota immediately. Thus, performing FMT during UC flare-up might result in only a transient effect. Moreover, a large number of microbial antigens on the inflamed mucosa might have detrimental effects. ${ }^{1}$ Sood et al. ${ }^{9}$ noted that patients aged $<40$ years, females, those treated early in the disease course or having a mild disease had better outcomes, but none of these showed statistical significance. In addition, the rate of clinical remission in patients who were on azathioprine was similar to that in those treated without azathioprine. Moayyedi et al. ${ }^{5}$ reported that subjects under immunosuppression achieved better outcomes (5 of 11 [46\%] in remission) than those solely on FMT (4 of 27 [15\%] in remission). This suggests that a combination of immunosuppressant therapy with FMT resulted in better outcomes. However, further validation is necessary about the effectiveness of pretreatment, including bowel lavage, pretreatment antibiotics, and combined drugs.

Unfortunately, Sood et al. ${ }^{9}$ did not perform fecal microbial analysis of the donors and recipients to evaluate the effect of FMT on the intestinal microbial change. The intrinsic characteristics of donor's microbiota could significantly affect the treatment outcome of FMT. ${ }^{1}$ Moayyedi et al. ${ }^{5}$ showed that most treatment successes were driven by a single donor, "donor B," whose microbiota was rich in members of the Lachnospiraceae family and Ruminococcus genus. However, despite a superdonor transplant, donor-recipient compatibilities might be more important for the outcome of FMT. ${ }^{2}$ Long-term safety after FMT for IBD is uncertain. FMT might cause possible transmission of infectious agents or the development of diseases associated with changes in the gut microbiota. ${ }^{1}$ Therefore, longterm follow-up of patients after FMT is necessary to answer questions concerning safety and future adverse events. Despite positive results from RCTs and the study by Sood et al., ${ }^{9}$ further well-designed large studies are necessary to confirm the efficacy and safety of FMT in routine clinical practice.

\section{FINANCIAL SUPPORT}

The authors received no financial support for the research, authorship, and/or publication of this article.

\section{CONFLICT OF INTEREST}

No potential conflict of interest relevant to this article was reported.

\section{AUTHOR CONTRIBUTION}

Writing and approval of final manuscript: Cho YS.

\section{REFERENCES}

1. Pigneur B, Sokol H. Fecal microbiota transplantation in inflammatory bowel disease: the quest for the holy grail. Mucosal Immunol 
2016;9:1360-1365.

2. Fairhurst NG, Travis SP. Why is it so difficult to evaluate faecal microbiota transplantation as a treatment for ulcerative colitis? Intest Res 2018;16:209-215.

3. Vaughn BP, Rank KM, Khoruts A. fecal microbiota transplantation: current status in treatment of GI and liver disease. Clin Gastroenterol Hepatol 2019;17:353-361.

4. Narula N, Kassam Z, Yuan Y, et al. Systematic review and metaanalysis: fecal microbiota transplantation for treatment of active ulcerative colitis. Inflamm Bowel Dis 2017;23:1702-1709.

5. Moayyedi P, Surette MG, Kim PT, et al. Fecal microbiota transplantation induces remission in patients with active ulcerative colitis in a randomized controlled trial. Gastroenterology 2015; 149:102-109.e6.

6. Rossen NG, Fuentes S, van der Spek MJ, et al. Findings from a randomized controlled trial of fecal transplantation for patients with ulcerative colitis. Gastroenterology 2015;149:110-118.e4.

7. Costello SP, Waters O, Bryant RV, et al. Short duration, low intensity, pooled fecal microbiota transplantation induces remission in patients with mild-moderately active ulcerative colitis: a randomised controlled trial. Gastroenterology 2017;152:S198-S199.

8. Paramsothy S, Kamm MA, Kaakoush NO, et al. Multidonor intensive faecal microbiota transplantation for active ulcerative colitis: a randomised placebo-controlled trial. Lancet 2017;389:12181228.

9. Sood A, Mahajan R, Juyal G, et al. Efficacy of fecal microbiota therapy in steroid dependent ulcerative colitis: a real world intention-to-treat analysis. Intest Res 2019;17:78-86.

10. Mizuno S, Nanki K, Matsuoka K, et al. Single fecal microbiota transplantation failed to change intestinal microbiota and had limited effectiveness against ulcerative colitis in Japanese patients. Intest Res 2017;15:68-74. 